\title{
Characterization of New ATM Deletion Associated with Hereditary Breast Cancer
}

\author{
Sandra Parenti ${ }^{1,2,+}$, Claudio Rabacchi ${ }^{1,3, \dagger}$, Marco Marino ${ }^{4,+}$, Elena Tenedini ${ }^{4}$, Lucia Artuso ${ }^{4}$, Sara Castellano ${ }^{3}$, \\ Chiara Carretta ${ }^{2} \mathbb{D}$, Selene Mallia ${ }^{2}$, Laura Cortesi ${ }^{5}$, Angela Toss ${ }^{5} \mathbb{D}$, Elena Barbieri ${ }^{5}$, Rossella Manfredini ${ }^{2}$, \\ Mario Luppi ${ }^{3,5}$, Tommaso Trenti ${ }^{4}$ and Enrico Tagliafico ${ }^{2, *(D)}$
}

1 Center for Genome Research, University of Modena and Reggio Emilia, 41125 Modena, Italy; sandra.parenti@unimore.it (S.P.); claudio.rabacchi@unimore.it (C.R.)

2 Centre for Regenerative Medicine, University of Modena and Reggio Emilia, 41125 Modena, Italy; chiara.carretta@unimore.it (C.C.); selene.mallia@unimore.it (S.M.); rossella.manfredini@unimore.it (R.M.)

3 Department of Medical and Surgical Sciences, University of Modena and Reggio Emilia, 41124 Modena, Italy; sara.castellano@unimore.it (S.C.); mario.luppi@unimore.it (M.L.)

4 Department of Laboratory Medicine and Pathology, Diagnostic Hematology and Clinical Genomics Unit, Modena University Hospital, 41124 Modena, Italy; marino.marco@aou.mo.it (M.M.); tenedini.elena@aou.mo.it (E.T.); artuso.lucia@aou.mo.it (L.A.); t.trenti@ausl.mo.it (T.T.)

5 Department of Oncology and Hematology, University Hospital of Modena, 41124 Modena, Italy; cortesi.laura@aou.mo.it (L.C.); angela.toss@unimore.it (A.T.); barbieri.elena@aou.mo.it (E.B.)

* Correspondence: enrico.tagliafico@unimore.it

+ These authors contributed equally to this work.

Citation: Parenti, S.; Rabacchi, C.; Marino, M.; Tenedini, E.; Artuso, L.; Castellano, S.; Carretta, C.; Mallia, S.; Cortesi, L.; Toss, A.; et al. Characterization of New ATM Deletion Associated with Hereditary Breast Cancer. Genes 2021, 12, 136. https://doi.org/10.3390/genes12020136

Received: 22 December 2020

Accepted: 18 January 2021

Published: 21 January 2021

Publisher's Note: MDPI stays neutral with regard to jurisdictional claims in published maps and institutional affiliations.

Copyright: (c) 2021 by the authors. Licensee MDPI, Basel, Switzerland. This article is an open access article distributed under the terms and conditions of the Creative Commons Attribution (CC BY) license (https:// creativecommons.org/licenses/by/ $4.0 /)$.

\begin{abstract}
Next-generation sequencing (NGS)-based cancer risk screening with multigene panels has become the most successful method for programming cancer prevention strategies. ATM germ-line heterozygosity has been described to increase tumor susceptibility. In particular, families carrying heterozygous germ-line variants of ATM gene have a 5- to 9-fold risk of developing breast cancer. Recent studies identified ATM as the second most mutated gene after CHEK2 in BRCA-negative patients. Nowadays, more than 170 missense variants and several truncating mutations have been identified in ATM gene. Here, we present the molecular characterization of a new ATM deletion, identified thanks to the CNV algorithm implemented in the NGS analysis pipeline. An automated workflow implementing the SOPHiA Genetics' Hereditary Cancer Solution (HCS) protocol was used to generate NGS libraries that were sequenced on Illumina MiSeq Platform. NGS data analysis allowed us to identify a new inactivating deletion of exons 19-27 of ATM gene. The deletion was characterized both at the DNA and RNA level.
\end{abstract}

Keywords: next-generation sequencing; breast cancer; ATM; homologous recombination repair; hereditary cancer syndromes; clinical genomics; molecular diagnostics

\section{Introduction}

Breast cancer is one of the most common tumors in the female population, and 5-10\% of cases arise as hereditary breast cancer (HBC) [1]. The assessment of the hereditary cancer risk that can be obtained following the mutational study with next-generation sequencing (NGS)-based multigene panels can allow the choice of the best risk prevention program and has become the most successful method for programming cancer prevention [2-4]. Although $B R C A 1$ and $B R C A 2$ remain the most frequently mutated genes, mutations in other DNA repair genes account for a significant fraction $(\sim 25 \%)$ of HBC cases [5]. ATM germ-line heterozygosity occurs in about $1 \%$ of the population and has been described to increase tumor susceptibility. In particular, families carrying heterozygous germ-line variants of $A T M$ gene have a 5- to 9-fold risk of developing breast cancer, particularly in women younger than 50 years [6]. Approximately, 3\% of the families affected by hereditary breast and ovarian cancer harbors a loss-of-function ATM mutation [7]. Recent 
studies identified ATM as the second most mutated gene after CHEK2 in BRCA-negative patients [8]. In particular, the cumulative risk of breast cancer by age 50 was estimated for ATM heterozygous mutation carriers to be $6.02 \%$, while by age 80 , was estimated to be $32.83 \%$ [9]. Nowadays, more than 170 missense variants and several truncating mutations have been identified in ATM gene [8]. Few data are available on the deletions and rearrangements of the ATM gene, especially since not all analytical pipelines of the NGS data implement specific algorithms for predicting CNVs. The artificial intelligence-based NGS data analysis platform, SOPHiA DDM software, incorporates a specific algorithm to study CNVs from NGS data generated using the SOPHiA Genetics' Hereditary Cancer Solution (HCS) kit. Here, we present the molecular validation and characterization both at DNA and RNA level of a new ATM deletion identified with diagnostic NGS for hereditary cancer risk screening.

\section{Materials and Methods}

\subsection{Subjects}

Written informed consent was obtained from all subjects. Total genomic DNA was extracted from blood samples using Maxwell 16 Blood DNA Purification Kit and Maxwell 16 MDx AS3000 instrument (Promega, Madison, WI, USA).

\subsection{NGS Sequencing}

Two hundred nanograms of genomic DNA has been processed with the Hereditary Cancer Solution (HCS) kit (SOPHiA GENETICS, Saint-Sulpice, Switzerland). The capturebased target enrichment of 26 cancer related genes (ATM, APC, BARD1, BRCA1, BRCA2, BRIP1, CDH1, CHEK2, EPCAM, FAM175A, MLH1, MRE11A, MSH2, MSH6, MUTYH, NBN, PALB2, PIK3CA, PMS2, PMS2C, PTEN, RAD50, RAD51C, RAD51D, STK11, TP53, XRCC2) and the library construction protocols were carried out exclusively with the automated procedure implemented on the STARlet platform (Hamilton Company, Reno, NV, USA). Library quantification was carried out with fluorometric quantitation using Qubit dsDNA High Sensitivity kit (Thermofisher Scientific, Waltham, MA, USA). Quality control was performed by analyzing the profile of each sample obtained with Bioanalyzer DNA 1000 (Agilent Technologies, Santa Clara, CA, USA).

Sequencing was achieved onto a 600-cycle format V3 flow-cell, via Illumina MiSeq DX (Illumina, San Diego, CA, USA) platform according to their and SOPHiA Genetics' protocols. Sequencing data were processed for single nucleotide variants (SNVs), indels, and copy number variations (CNVs) via the SOPHiA DDM platform based on SOPHiA Artificial Intelligence (AI).

\subsection{Multiplex Ligation-Dependent Probe Amplification (MLPA) Analysis}

One hundred nanograms of genomic DNA of the proband was analyzed for CNVs detection in the ATM gene with SALSA MLPA P041 and P042 probemix, (MRC Holland, Amsterdam, The Netherlands) according to manufacturer's instructions on ABIPrism 3130XL Genetic Analyzer (Thermo Fisher Scientific). The data were analyzed with Coffalyzer software (MRC Holland).

\subsection{Long-Range PCR of the Gene Region Including the Deletion}

The deletion, detected by NGS sequencing and MLPA, was confirmed with long-range PCR using the Fast Start PCR Master Mix (Roche, Penzberg, Germany). FastPCR Software (Primer Digital Ltd., Helsinki, Finland) was used to design the following primers:

ATM-e18-4FW: 5'-GTGTAACTACTGCTCAGACCAA-3'

ATM-e28-8R: 5'-CAGACCAATACTGTGTCCTTTAGGGCA-3'

DNA was amplified using the following PCR conditions: an initial denaturation at 94 ${ }^{\circ} \mathrm{C}$ for $3^{\prime}$, followed by 10 cycles at $94^{\circ} \mathrm{C}$ for $20^{\prime \prime}, 59^{\circ} \mathrm{C}$ for $30^{\prime \prime}, 68^{\circ} \mathrm{C}$ for $7^{\prime}$, and by other 20 cycles at $94^{\circ} \mathrm{C}$ for $20^{\prime \prime}, 59^{\circ} \mathrm{C}$ for $30^{\prime \prime}$ and $68^{\circ} \mathrm{C}$ for $7^{\prime}$ with an increase of $10^{\prime \prime}$ for every PCR cycle, and a final extension at $68^{\circ} \mathrm{C}$ for $13^{\prime}$. 


\subsection{Characterization of the Deletion Breakpoint}

The PCR product was sequenced with ABIPrism 3130XL Genetic Analyzer (Thermo Fisher Scientific), using the following primers:

ATM-e18-10FW: 5'-CTACCAAATCCCTCCACCTGCAT-3'

ATM-E28-7R: 5'-GAGCAGGATCCAAATCCCTAACAGAGT-3'

The nucleotide sequence was compared to wild-type ATM genomic sequence [LRG_135, NG_009830.1]. Intronic repeated sequences were found with Repeat Masker software (http:/ / www.repeatmasker.org/cgi-bin/WEBRepeatmasker).

\subsection{Analysis of ATM mRNA}

Total RNA was extracted with Maxwell 16 Total RNA Purification Kit and Maxwell 16 MDx AS3000 instrument (Promega). In total, 500 ng of total RNA was reverse transcribed using MMLV Reverse Transcriptase (Thermo Fisher Scientific) as previously described [10]. PCR amplification of the gene region including the deletion was performed as described above. Then, the PCR fragment harboring the deletion was extracted from agarose gel and sequenced as previously described [11]. The nucleotide sequence of the amplified cDNA fragment was compared to the wild-type sequence of ATM mRNA [NM_000051.3].

\section{Results}

\subsection{Identification of Genomic Variant by NGS Analysis}

The proband, belonging to a family of Italian ancestry, had been referred for genetic testing based on a family history of breast cancer at early onset. She received a diagnosis of unilateral breast cancer HER2+, ER+ and PR - at age 34. Her mother was affected by unilateral hormone receptors positive (HER2,$+ \mathrm{ER}+, \mathrm{PR}+$ ) breast cancer, diagnosed at the age of 45 years, with metastases in axillary lymph nodes. The proband was previously tested for the mutational status of only $B R C A 1$ and $B R C A 2$ genes and reported as negative for pathogenic variants. In order to identify any further variants that could explain the phenotype, the proband was analyzed using the CE-IVD Hereditary Cancer Solution (HCS) assay by SOPHiA Genetics. The results revealed a wide heterozygous deletion of exons from 19 to 27 of $A T M$ gene.

\subsection{MLPA Analysis and Breakpoint Characterization}

In order to confirm the deletion of the region spanning from exon 19 to exon 27 of the ATM gene, MLPA analysis was performed on the genomic DNA of the proband with two different mixes of probes in order to examine the whole gene. The results confirmed this deletion in heterozygous state (Figure S1). To further characterize the deletion, we used a long-range PCR design to amplify the breakpoint region in proband DNA and, both in her mother and sister, for verifying the segregation. This assay confirmed the presence of the deletion, showing a $2.5 \mathrm{~Kb}$ PCR product in proband and in her mother (Figure 1). In order to explain the possible causal mechanism of the deletion, we analyzed the sequence using the Repeat Masker software for the identification of interspersed repeats and low complexity DNA sequences. The output revealed several repetitive genomic elements, as ALU sequences, in particular in intron 18. To characterize in detail the exact breakpoint, we sequenced the PCR amplicon by Sanger sequencing, revealing a small six-nucleotide element, GGCTCA, overlapping the two introns involved in the rearrangement (Figure 2) and allowing the accurate description of the deletion at DNA level (c.2838+2162_4110-292del). 


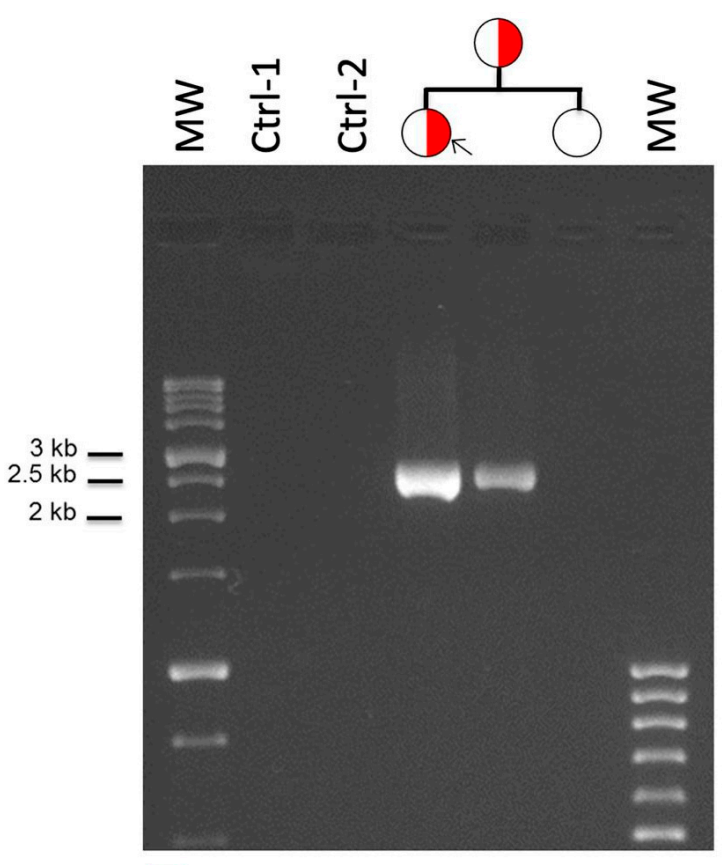

ATM c.2838+2162_4110-292del

Figure 1. Breakpoint characterization of ATM exon 19-27 deletion: long-range PCR confirmed the presence of the deletion, showing a $2.5 \mathrm{~Kb}$ PCR product in proband DNA. The analysis of other family members confirms the presence of the deletion in proband's mother (lanes 4 and 5). Two DNAs from healthy subjects were amplified as control (lanes 2 and 3). Molecular weight (MW) markers are loaded in lanes 1 and 7.

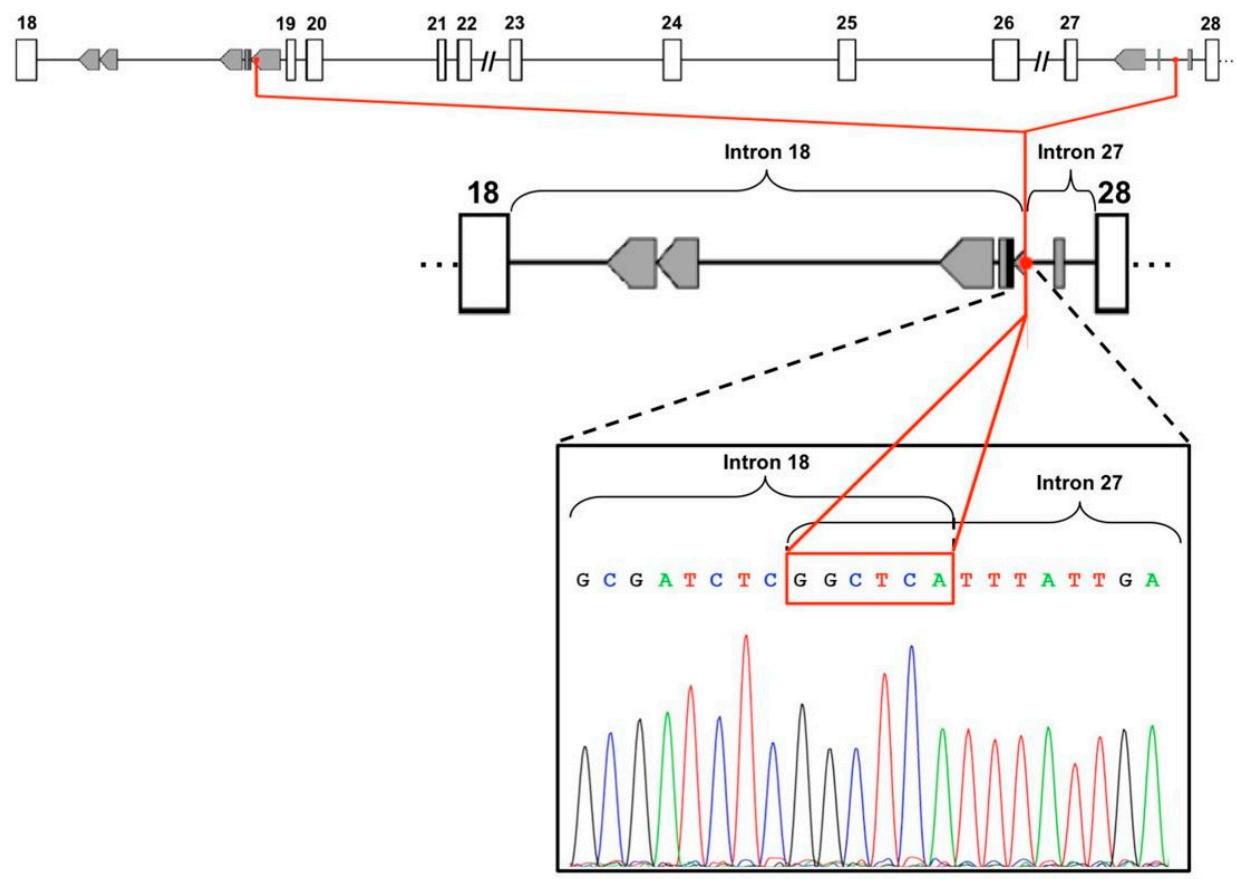

Figure 2. Analysis of repetitive genomic elements and Sanger sequencing: upper and middle part of the figure show the analysis of genomic sequences performed through Repeat Masker software that revealed several repetitive genomic elements, in particular ALU sequences (in gray boxes), in intron 18. Lower part of the figure, Sanger sequencing of the $2.5 \mathrm{~Kb}$ PCR product shows the breakpoint region of the rearrangement, characterized by a small six-nucleotide element, GGCTCA, overlapping the two introns. 


\subsection{Characterization of the mRNA Sequence}

To characterize the RNA transcript derived from the deleted allele, we reversetranscribed the proband's mother and sister RNA. Unfortunately, the proband's RNA was no longer available. The cDNA was then amplified and the gene region carrying the deletion was sequenced. As shown in Figure 3A, the mother cDNA originated both the wild-type allele (1500 bp) and the mutated one (200 bp). In the mutated isoform, exon 28 is joined to exon 18 (Figure 3B). This variant disrupts the reading frame of the mRNA generating a premature stop codon predicted to encode for a truncated protein of 952 aa, instead of the wild-type 3056 aa ATM protein (p.Tyr947Glyfs*7).

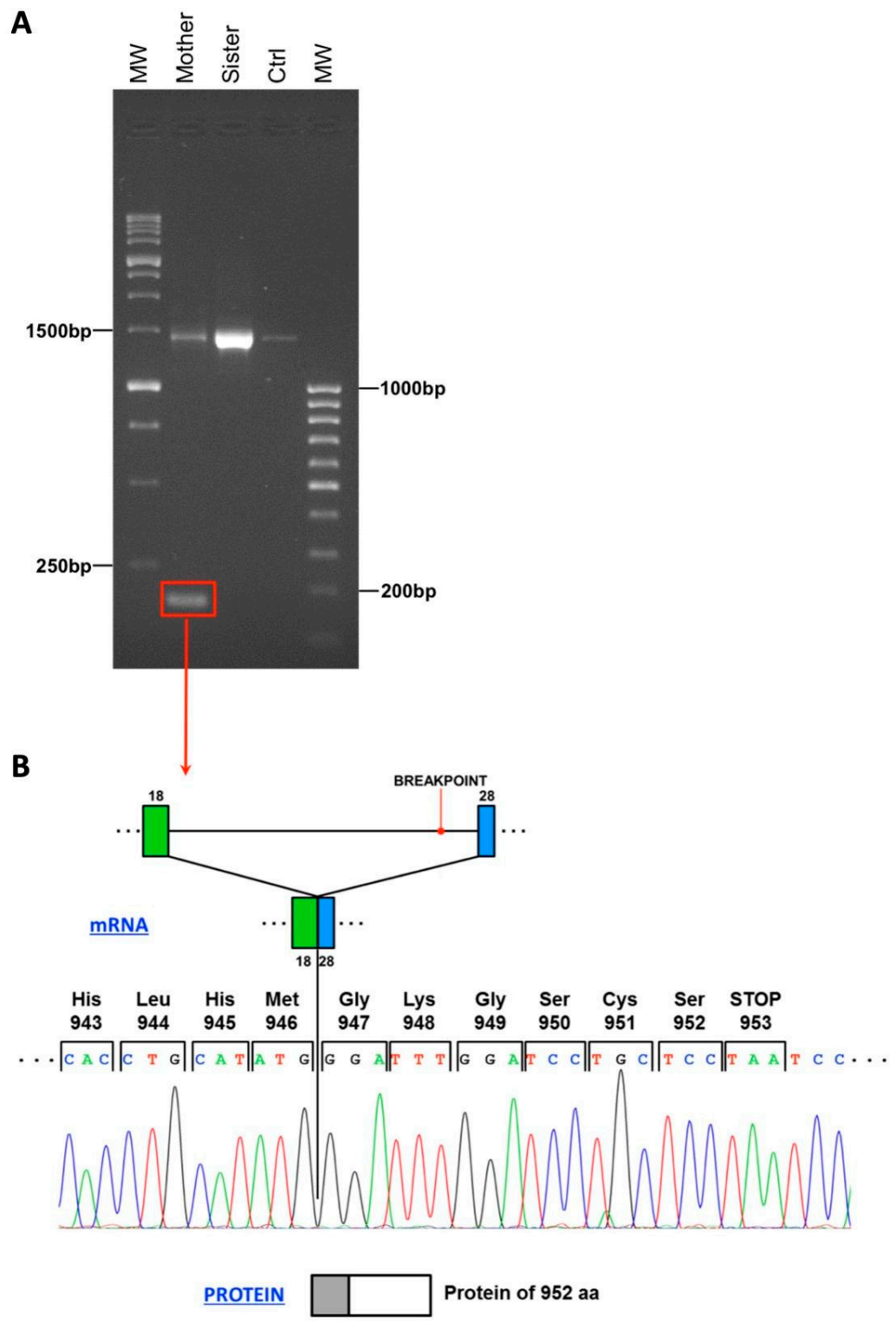

Figure 3. (A) Characterization of mRNA sequence resulting from the deletion: RNA derived from proband's mother showed a 200 bp PCR product represented in Panel A that underwent Sanger sequencing, displayed in Panel B. Proband's sister shows only the wild-type form of the transcript. (B) This analysis allowed us to demonstrate that this variant generates a premature stop codon that encodes for a truncated protein of 952 aa (grey box), instead of the wild-type 3056 aa ATM protein (grey+white boxes). 


\section{Discussion}

The characterization of the mutational status of genes involved in homologous recombination repair pathway is absolutely the most important approach for designing the prevention strategies for inherited cancer syndromes. A large number of missense and truncating ATM variants have been detected in BRCA1- or BRCA2-negative patients [8].

SOPHiA Genetics HCS gene panel allowed us to detect a novel ATM CNV in a BRCAnegative HBC patient. The deletion of exons 19 to 27 of the ATM gene was detected in an Italian woman who received a diagnosis of unilateral breast cancer at the age of 34 . Her mother had unilateral receptor-positive breast cancer, at the age of 45 , with axillary lymph node involvement. The NGS detected CNV was firstly validated by MLPA. Afterward, long-range PCR and Sanger sequencing were used in order to characterize the breakpoint at DNA level (c.2838+2162_4110-292del) in proband and to study the segregation also in her mother and in her sister.

A further characterization at RNA level on the proband's mother and sister identified the presence of both the wild-type and the mutant allele in the mother's sample. In the mutated isoform, exon 28 is joined to exon 18, disrupting the mRNA reading frame and generating a premature stop codon. The predicted aberrant ATM protein consists of 952 amino acids instead of 3056 amino acids of wild-type ATM. This abnormal protein lacks the domain involving the amino acid positions 1373-1382, required for c-Abl protein interaction and crucial to mediate cell cycle arrest in G1 phase [12]. In addition, at least three other important domains are deleted from the ATM protein, such as FAT (FRAP-ATM-TRRAP), PIKK (phosphatidylinositol 3-kinase-related kinase domain) and FATC (FAT C-terminal domain) domains, mediating the majority of ATM functions $[13,14]$.

Supplementary Materials: The following are available online at https:/ / www.mdpi.com/2073-442 5/12/2/136/s1, Figure S1: MLPA validation of ATM deletion. The two upper panels show MLPA analysis performed through SALSA MLPA P041 and P042 probemix on a negative control. The two lower panels display the same analysis performed on the proband sample, confirming the presence of a deletion spanning from exon 19 to 27 of ATM gene.

Author Contributions: Conceptualization, E.Ta.; methodology, E.Ta, S.P., C.R., E.T. (Elena Tenedini), M.M., L.A. and S.C.; investigation, S.P., C.R., E.T. (Elena Tenedini), M.M., L.A., S.C., C.C., S.M., L.C., A.T. and E.B.; writing-original draft preparation, S.P., C.R., M.M and E.T. (Enrico Tagliafico); writing-review and editing, R.M., M.L. and T.T.; supervision, E.T. (Enrico Tagliafico). All authors have read and agreed to the published version of the manuscript.

Funding: This research received no external funding.

Institutional Review Board Statement: Ethical review and approval were waived for this work by following the guidelines of our Regional Ethics Committee which reports that the evaluation of a single case report or case series (up to 5 cases) whose sole purpose is reporting, i.e., the purpose of which is to inform the scientific community by describing some unusual or new cases, is not within the competence of the Ethics Committee.

Informed Consent Statement: Informed consent was obtained from all subjects involved in the study.

Conflicts of Interest: The authors declare no conflict of interest.

\section{References}

1. Harbeck, N.; Penault-Llorca, F.; Cortes, J.; Gnant, M.; Houssami, N.; Poortmans, P.; Ruddy, K.; Tsang, J.; Cardoso, F. Breast cancer. Nat. Rev. Dis Primers 2019, 5, 66. [CrossRef] [PubMed]

2. Neben, C.L.; Zimmer, A.D.; Stedden, W.; van den Akker, J.; O'Connor, R.; Chan, R.C.; Chen, E.; Tan, Z.; Leon, A.; Ji, J.; et al. Multi-Gene Panel Testing of 23,179 Individuals for Hereditary Cancer Risk Identifies Pathogenic Variant Carriers Missed by Current Genetic Testing Guidelines. J. Mol. Diagn. 2019, 21, 646-657. [CrossRef] [PubMed]

3. Susswein, L.R.; Marshall, M.L.; Nusbaum, R.; Vogel Postula, K.J.; Weissman, S.M.; Yackowski, L.; Vaccari, E.M.; Bissonnette, J.; Booker, J.K.; Cremona, M.L.; et al. Pathogenic and likely pathogenic variant prevalence among the first 10,000 patients referred for next-generation cancer panel testing. Genet. Med. 2016, 18, 823-832. [CrossRef] [PubMed] 
4. Crawford, B.; Adams, S.B.; Sittler, T.; van den Akker, J.; Chan, S.; Leitner, O.; Ryan, L.; Gil, E.; van't Veer, L. Multi-gene panel testing for hereditary cancer predisposition in unsolved high-risk breast and ovarian cancer patients. Breast Cancer Res. Treat. 2017, 163, 383-390. [CrossRef] [PubMed]

5. Cobain, E.F.; Milliron, K.J.; Merajver, S.D. Updates on breast cancer genetics: Clinical implications of detecting syndromes of inherited increased susceptibility to breast cancer. Semin. Oncol. 2016, 43, 528-535. [CrossRef] [PubMed]

6. Choi, M.; Kipps, T.; Kurzrock, R. ATM Mutations in Cancer: Therapeutic Implications. Mol. Cancer Ther. 2016, 15, 1781-1791. [CrossRef] [PubMed]

7. Renault, A.L.; Mebirouk, N.; Fuhrmann, L.; Bataillon, G.; Cavaciuti, E.; Le Gal, D.; Girard, E.; Popova, T.; La Rosa, P.; Beauvallet, J.; et al. Morphology and genomic hallmarks of breast tumours developed by ATM deleterious variant carriers. Breast Cancer Res. 2018, 20, 28. [CrossRef] [PubMed]

8. Jerzak, K.J.; Mancuso, T.; Eisen, A. Ataxia-telangiectasia gene (ATM) mutation heterozygosity in breast cancer:A narrative review. Curr. Oncol. 2018, 25, e176-e180. [CrossRef] [PubMed]

9. Marabelli, M.; Cheng, S.C.; Parmigiani, G. Penetrance of ATM Gene Mutations in Breast Cancer: A Meta-Analysis of Different Measures of Risk. Genet. Epidemiol 2016, 40, 425-431. [CrossRef] [PubMed]

10. Vignudelli, T.; Selmi, T.; Martello, A.; Parenti, S.; Grande, A.; Gemelli, C.; Zanocco-Marani, T.; Ferrari, S. ZFP36L1 negatively regulates erythroid differentiation of CD34+ hematopoietic stem cells by interfering with the Stat5b pathway. Mol. Biol. Cell 2010, 21, 3340-3351. [CrossRef] [PubMed]

11. Marino, M.; Rabacchi, C.; Simone, M.L.; Medici, V.; Cortesi, L.; Calandra, S. A novel deletion of BRCA1 gene that eliminates the ATG initiation codon without affecting the promoter region. Clin. Chim. Acta. 2009, 403, 249-253. [CrossRef] [PubMed]

12. Shafman, T.; Khanna, K.K.; Kedar, P.; Spring, K.; Kozlov, S.; Yen, T.; Hobson, K.; Gatei, M.; Zhang, N.; Watters, D.; et al. Interaction between ATM protein and c-Abl in response to DNA damage. Nature 1997, 387, 520-523. [CrossRef] [PubMed]

13. Weber, A.M.; Drobnitzky, N.; Devery, A.M.; Bokobza, S.M.; Adams, R.A.; Maughan, T.S.; Ryan, A.J. Phenotypic consequences of somatic mutations in the ataxia-telangiectasia mutated gene in non-small cell lung cancer. Oncotarget 2016, 7, 60807-60822. [CrossRef] [PubMed]

14. Kozlov, S.V.; Graham, M.E.; Jakob, B.; Tobias, F.; Kijas, A.W.; Tanuji, M.; Chen, P.; Robinson, P.J.; Taucher-Scholz, G.; Suzuki, K.; et al. Autophosphorylation and ATM activation:Additional sites add to the complexity. J. Biol Chem 2011, 286, 9107-9119. [CrossRef] [PubMed] 